\title{
Discovery of novel plasma protein biomarkers to predict imminent cystic fibrosis pulmonary exacerbations using multiple reaction monitoring mass spectrometry
}

\author{
Bradley S Quon, ${ }^{1,2}$ Darlene L Y Dai, ${ }^{3}$ Zsuzsanna Hollander, ${ }^{3}$ Raymond T Ng, ${ }^{3,4}$ \\ Scott J Tebbutt, ${ }^{1,2,3}$ S F Paul Man, ${ }^{1,2}$ Pearce G Wilcox, ${ }^{1,2}$ Don D Sin ${ }^{1,2}$
}

- Additional material is published online only. To view please visit the journal online (http://dx.doi.org/10.1136/ thoraxjn-2014-206710)

For numbered affiliations see end of article.

\section{Correspondence to} Dr Bradley S Quon, Division of Respiratory Medicine, Department of Medicine, University of British Columbia, 1081 Burrard Street, 8B Providence Wing, Vancouver, British Columbia, Canada V6z 1Y6; bradley.quon@hli.ubc.ca

A portion of the data was presented in abstract form at the 2014 North American Cystic Fibrosis Conference on 9-11 October 2014 in Atlanta, Georgia.

Received 17 December 2014 Revised 24 February 2015 Accepted 26 February 2015 Published Online First 16 March 2015

\section{SLinked}

- http://dx.doi.org/10.1136/ thoraxjnl-2014-206750

CrossMark

To cite: Quon BS, Dai DLY, Hollander $Z$, et al. Thorax 2016:71:216-222.

\section{ABSTRACT}

Background Despite the significant morbidity and mortality related to pulmonary exacerbations in cystic fibrosis (CF), there remains no reliable predictor of imminent exacerbation.

Objective To identify blood-based biomarkers to predict imminent ( $<4$ months from stable blood draw) CF pulmonary exacerbations using targeted proteomics.

Methods 104 subjects provided plasma samples when clinically stable and were randomly split into discovery $(n=70)$ and replication $(n=34)$ cohorts. Multiple reaction monitoring mass spectrometry (MRM-MS) was used to measure 117 peptides (79 proteins) from plasma. Plasma proteins with differential abundance between subjects who did versus did not develop an imminent exacerbation were analysed and proteins with fold difference $>1.5$ between the groups were included in an MRM-MS classifier model to predict imminent exacerbations. Performance characteristics were compared with clinical predictors and candidate plasma protein biomarkers.

Results Six proteins were included in the final MRMMS protein panel. The area under the curve (AUC) for the prediction of imminent exacerbations was highest for the MRM-MS protein panel (AUC 0.74) in comparison to $\mathrm{FEV}_{1} \%$ predicted (AUC 0.55) and the top candidate plasma protein biomarkers, including C-reactive protein (AUC 0.61) and interleukin-6 (AUC 0.60). The MRM-MS protein panel performed similarly in the replication cohort (AUC 0.73).

Conclusions Using MRM-MS, a six-protein panel measured from plasma can distinguish individuals with versus without an imminent exacerbation. With further replication and assay development, this biomarker panel may be clinically applicable for prediction of exacerbations in individuals with CF.

\section{INTRODUCTION}

Among the most significant clinical events for individuals with cystic fibrosis (CF) are pulmonary exacerbations. These are important life events because they can lead to permanent loss in lung function, ${ }^{1} 2$ reduced quality of life ${ }^{34}$ and increased risk of death. ${ }^{5}{ }^{6}$ Despite notable improvements in other important health outcomes for individuals with CF over the past two decades (such as pulmonary function and nutritional status),

\section{Key messages}

What is the key question?

- Can targeted proteomics be used to discover blood-based biomarkers to predict imminent pulmonary exacerbations ( $<4$ months) in cystic fibrosis (CF)?

\section{What is the bottom line?}

- Using multiple reaction monitoring mass spectrometry, a six-protein biomarker panel was identified and validated to predict imminent CF pulmonary exacerbations, outperforming clinical predictors and candidate protein biomarkers.

\section{Why read on?}

- A simple, minimally invasive blood test can be used to predict imminent CF pulmonary exacerbations and this biomarker might be applied clinically to develop pre-emptive strategies to prevent exacerbations or mitigate their potential impact.

exacerbations continue to be common. Based on the CF Foundation Patient Registry Report, 35\% of patients experienced at least one exacerbation requiring intravenous antibiotics across the USA in 2012..$^{7}$ Given the potential devastating consequences of exacerbations, preventing these events remains a management priority for CF physicians. Fortunately, many of the maintenance respiratory therapies including mucolytics, inhaled antibiotics and oral azithromycin approved for use in CF are beneficial in preventing exacerbations. ${ }^{8}$

Currently, CF clinicians do not have a reliable means to predict which of their patients will develop an exacerbation prior to their next clinic visit. With this uncertainty in predicting exacerbations, it remains challenging for physicians to decide if and when to intensify therapies. While previous studies have identified prior exacerbation history and current lung function as predictors of future exacerbation risk, these studies were focused on subgroups of patients harbouring multidrug-resistant organisms ${ }^{9}$ and those not 
receiving guideline recommended therapies. ${ }^{10}$ Furthermore, these clinical variables are unlikely to reflect the current biological status of the patient sufficiently to guide day-to-day treatment decisions for personalised medicine.

High-throughput '-omics' technologies (eg, proteomics, transcriptomics) are now available that can measure the relative abundances of different molecular targets (eg, protein, RNA) for assessment as biomarkers to predict risk or disease. ${ }^{11}$ Multiple reaction monitoring mass spectrometry (MRM-MS) is a targeted proteomics technology that offers the advantage of precise and simultaneous measurement of at least 100 protein targets per sample. ${ }^{12}$ To our knowledge, MRM-MS has yet to be applied to biomarker discovery in CF. Blood represents an amenable tissue to apply proteomics technology for biomarker discovery in CF because it is relatively easy to obtain and represents the 'pipeline' of the immune system. ${ }^{13}$ The detection of a circulating protein profile indicative of an impending exacerbation (prior to a patient being symptomatic) has the potential to transform patient care as it might enable CF physicians to predict exacerbations. The objectives of this study were to (1) use MRM-MS to identify a novel biomarker panel of proteins in the blood to predict imminent pulmonary exacerbations in CF and (2) compare the performance of the MRM-MS protein panel to established clinical predictors of exacerbations and other candidate protein biomarkers previously investigated in the context of exacerbations.

\section{METHODS}

\section{Study population}

We enrolled adults with an established diagnosis of CF based on standard criterion ${ }^{14}$ and without prior solid organ transplantation from the St. Paul's Hospital Adult Cystic Fibrosis Clinic (Vancouver, British Columbia, Canada) between April 2009 and April 2013. Consenting participants were included in this study if they had at least one plasma sample obtained when clinically stable during a routine clinic visit, defined by the absence of an exacerbation in the 4 weeks leading up to and including the enrolment clinic visit. Exacerbations were defined as a change in clinical parameters (based on the modified Fuchs criteria ${ }^{15}$ ) requiring additional treatment (oral or intravenous antibiotics), based on a consensus clinical trial definition recommended by the EuroCareCF Working Group. ${ }^{16}$ Patients who were receiving active treatment for non-tuberculous mycobacterial disease or allergic bronchopulmonary aspergillosis, or were taking systemic corticosteroids were excluded. In total, 104 of 185 potentially eligible patients with CF met our inclusion/exclusion criteria and provided informed consent for blood collection (figure 1). If multiple longitudinal stable plasma samples were available for individual patients, the first stable sample was included in this analysis.

\section{Sample collection}

Venous blood samples were collected in EDTA tubes from clinically stable participants using standard operating procedures. Samples were immediately stored on ice, processed for plasma and stored within $2 \mathrm{~h}$ at $-80^{\circ} \mathrm{C}$ until thawing for batched analysis.

\section{Clinical data collection}

Chart review was performed to obtain baseline clinical characteristics to predict exacerbations. The following clinical variables were collected corresponding to the time of stable blood draw: age, gender, CF transmembrane regulator genotype (F508del homozygous, heterozygous or other), body mass index
(BMI), $\mathrm{FEV}_{1} \%$ predicted, exacerbations requiring intravenous antibiotics in the prior year, CF-related diabetes, sputum microbiology and active use of chronic respiratory therapies (nebulised antibiotics and oral azithromycin). Spirometric data were obtained using standard techniques in accordance with guidelines from the American Thoracic Society. ${ }^{17}$ Charts were also reviewed to ascertain the timing of the first exacerbation (as defined above) following stable blood draw, and all participants had at least 6 months of follow-up data.

\section{MRM-MS proteomic analysis}

Plasma samples were sent to the University of Victoria (UVic) Genome BC Proteomics Centre (Victoria, British Columbia, Canada) for MRM-MS proteomic analysis. MRM-MS is a tandem MS (MS/MS) scan mode unique to triple quadruple MS instrumentation that monitors generation of a fragment ion by collision-induced dissociation (CID) from an isolated precursor ion. ${ }^{12}$ It is a highly sensitive and targeted proteomics approach that requires knowledge of the molecular weight of the analyte and its fragmentation behaviour under CID. The MRM-MS technique used by the UVic Proteomics Centre has been described in detail previously. ${ }^{18}{ }^{19}$ Briefly, the plasma sample is trypsin-digested overnight and then known concentrations of stable isotope-labelled reference peptides (unique to each protein of interest) are spiked into the digested sample as internal standards to enhance analyte detection confidence and measurement precision. The mixture is then separated via liquid chromatography and the eluted peptide fragments are ionised with an electrospray emitter. Using tandem MS, peptide ions are selected for fragmentation and fragment ions are selected for detection based on a user-specified list of targeted precursorproduct ion pairs (ie, transitions). Peptide concentrations are derived by measurement of the peak area ratios of one or more of the fragment ions from the labelled internal standard and the endogenous peptide. For this study, a bio-library consisting of 117 isotope-labelled reference peptides corresponding to 79 unique proteins (mostly involved in immunity, inflammation, coagulation and the acute phase response) were investigated (see online supplementary table S1).

\section{Candidate protein biomarker assays}

Candidate protein biomarkers were chosen based on a systematic review of the literature of blood-based biomarkers demonstrating promise for use in the context of CF pulmonary exacerbations ${ }^{20}$ and included plasma C-reactive protein (CRP), plasma interleukin-6 (IL-6), plasma soluble cluster of differentiation 14 (sCD14), serum myeloperoxidase (MPO) and plasma neutrophil elastase antiproteinase complex (NEAPC). All bloodbased candidate protein biomarkers were quantified using commercially available ELISAs except CRP, which was examined with MRM-MS alone as prior pilot work involving $30 \mathrm{CF}$ plasma samples demonstrated very strong correlation (Spearman's correlation $=0.96, \mathrm{p}<0.001$ ) in CRP measurements between the two techniques. The coefficient of variation for the IL-6 (R\&D Systems, Minneapolis, Minnesota, USA), sCD14 (R\&D Systems), MPO (R\&D Systems) and NEAPC (EMD Millipore, Billerica, Massachusetts, USA) ELISA kits were 3.1\%, $2.8 \%, 1.9 \%$ and $4.5 \%$, respectively.

\section{Outcome of interest}

A priori, our outcome of interest was the prediction of an imminent pulmonary exacerbation (as defined above) $<4$ months post-stable visit blood draw. A time frame of 4 months was chosen as this represents the average length of time between 


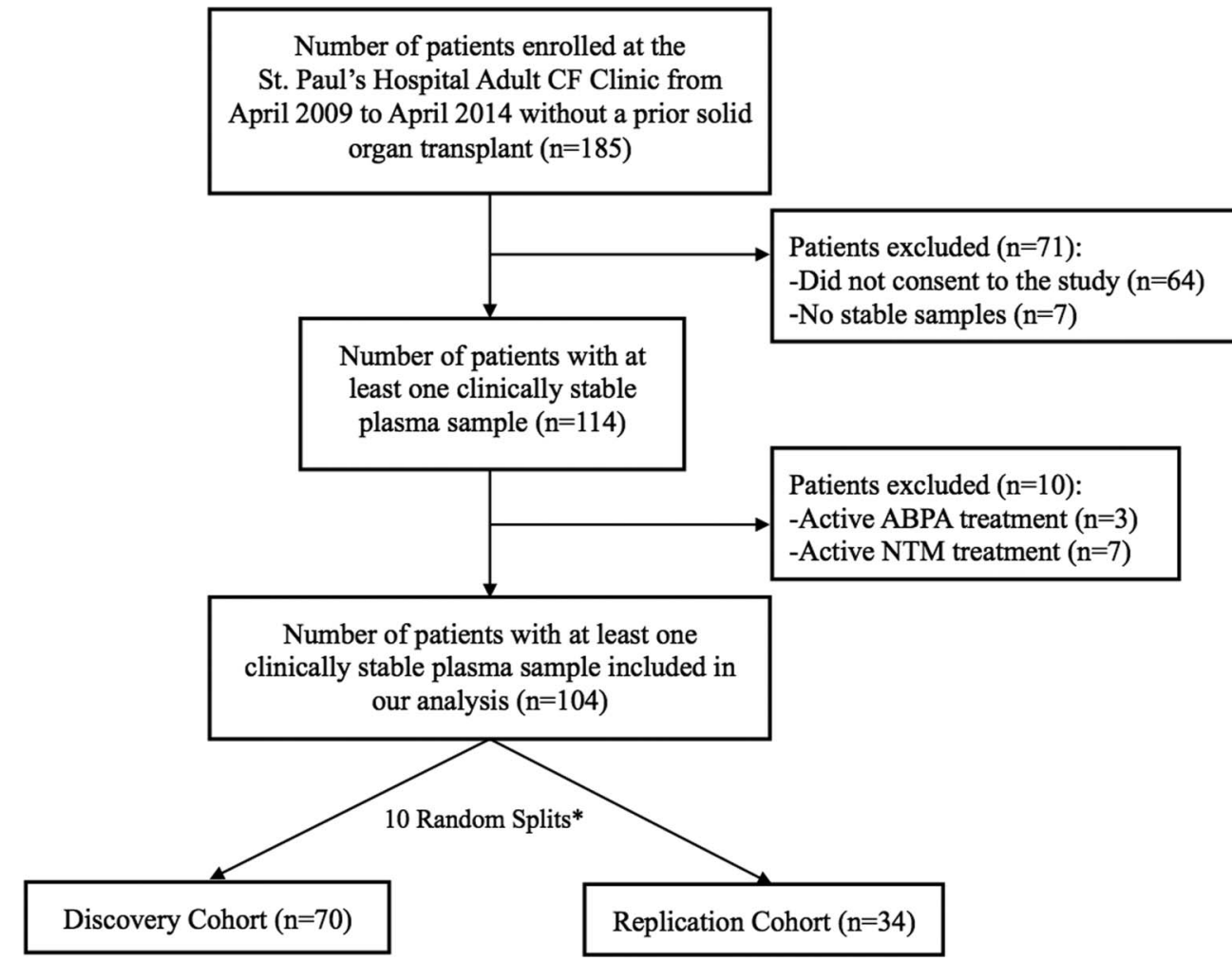

Figure 1 Flow diagram of cohort selection. * The random split resulting in the most balanced characteristics between discovery and replication cohorts was chosen for analysis. ABPA, allergic bronchopulmonary aspergillosis; CF, cystic fibrosis; NTM, non-tuberculous mycobacteria.

routine clinic visits for most patients attending our clinic. The ability of a biomarker to predict an exacerbation event prior to the next clinic visit provides the greatest opportunity to

Table 1 Characteristics and therapies of the discovery and replication cohorts

\begin{tabular}{|c|c|c|c|}
\hline Characteristic & $\begin{array}{l}\text { Discovery } \\
(n=70)\end{array}$ & $\begin{array}{l}\text { Replication } \\
(n=34)\end{array}$ & p Value \\
\hline Age (years) & $31.9 \pm 10.9$ & $35.4 \pm 11.9$ & 0.16 \\
\hline Male & $45(64 \%)$ & $23(68 \%)$ & 0.74 \\
\hline Genotype & & & 0.22 \\
\hline Homozygous F508del & $26(37 \%)$ & $12(35 \%)$ & \\
\hline Heterozygous F508del & $29(41 \%)$ & $19(56 \%)$ & \\
\hline Other & $15(21 \%)$ & $3(9 \%)$ & \\
\hline BMI $\left(\mathrm{kg} / \mathrm{m}^{2}\right)$ & $23.1 \pm 3.6$ & $23.9 \pm 3.6$ & 0.34 \\
\hline $\mathrm{FEV}_{1}$ (\% predicted) & $74.1 \pm 28.0$ & $69.9 \pm 23.8$ & 0.51 \\
\hline \multicolumn{4}{|l|}{ Sputum microbiology: } \\
\hline MSSA & $35(50 \%)$ & $18(53 \%)$ & 0.82 \\
\hline MRSA & $9(13 \%)$ & $3(9 \%)$ & 0.53 \\
\hline Pseudomonas aeruginosa & $32(46 \%)$ & $19(56 \%)$ & 0.26 \\
\hline Stenotrophomonas maltophilia & $2(3 \%)$ & $2(6 \%)$ & 0.52 \\
\hline Burkholderia cepacia complex & $8(11 \%)$ & $7(21 \%)$ & 0.24 \\
\hline CF-related diabetes & $21(30 \%)$ & $11(32 \%)$ & 0.91 \\
\hline $\begin{array}{l}\text { Exacerbation requiring } \\
\text { intravenous antibiotics in prior } \\
\text { year }\end{array}$ & $23(33 \%)$ & $11(32 \%)$ & 0.99 \\
\hline \multicolumn{4}{|l|}{ Current therapies } \\
\hline Inhaled antibiotics & $17(24 \%)$ & $8(24 \%)$ & 0.91 \\
\hline Oral azithromycin & $26(37 \%)$ & $13(38 \%)$ & 0.93 \\
\hline
\end{tabular}

intervene to prevent these events. In total, 43 (41\%) of 104 patients were diagnosed with an exacerbation $<4$ months following stable blood draw.

\section{Discovery and replication cohorts}

All 104 clinically stable patients with CF meeting our inclusion/ exclusion criteria were included in the analysis. The 104 patients were randomly split 10 times into two cohorts, discovery cohort (70 patients) and replication cohort (34 patients) (figure 1). Of these 10 splits, only the one that was most balanced on the clinical variables (table 1) was used for statistical analysis.

\section{Statistical analysis}

Preprocessing of the MRM-MS data involved several steps as described previously. ${ }^{21}$ Peptides that did not pass quality control metrics and those present in $<75 \%$ of the patient samples were eliminated from the analysis. If the peptide was not detected in a sample, it was replaced with half of the minimum detected peptide level. Relative ratios of endogenous to stable isotopelabelled peptide abundance were $\log _{2}$ transformed and summarised at the protein level to create protein abundance data.

Statistical analysis was performed using R (http://www. r-project.org), Bioconductor (http://www.bioconductor.org) and STATA V.12.0 (College Station, Texas, USA). Pearson's correlation analysis was conducted to examine the relationship between individual candidate protein biomarkers and proteins from the final MRM-MS protein panel. Candidate protein biomarker levels were compared between the groups of patients who did versus did not experience an exacerbation within 4 months of stable blood draw groups using the Student $t$ test. MRM-MS proteomic data were analysed for differential abundance between the two groups using robust-LIMMA ${ }^{22}$ (limma Bioconductor package). A random forest model was then 
Table 2 OR for individual clinical variables in the prediction of imminent cystic fibrosis (CF) pulmonary exacerbations (ie, $<4$ months post-stable visit)

\begin{tabular}{lll}
\hline Clinical predictor & OR (95\% Cl) & p Value \\
\hline Age (per year increase) & $1.01(0.95$ to 1.05$)$ & 0.80 \\
Male (vs. female) & $1.20(0.44$ to 3.24$)$ & 0.72 \\
Homozygous F508del & $1.24(0.47$ to 3.29$)$ & 0.67 \\
(vs non-homozygous F508del) & & \\
BMI (per kg/m² increase) & $0.87(0.75$ to 1.02$)$ & 0.08 \\
FEV ${ }_{1}$ (per \% predicted increase) & $0.99(0.97$ to 1.00$)$ & 0.13 \\
Sputum microbiology & & \\
$\quad$ MSSA & $0.79(0.31$ to 2.04$)$ & 0.63 \\
$\quad$ MRSA & $0.63(0.14$ to 2.75$)$ & 0.54 \\
$\quad$ Pseudomonas aeruginosa & $2.79(1.05$ to 7.40$)$ & 0.04 \\
$\quad$ Stenotrophomonas maltophilia & $1.34(0.08$ to 22.4$)$ & 0.84 \\
$\quad$ Burkholderia cepacia complex & $0.40(0.08$ to 2.16$)$ & 0.29 \\
CF-related diabetes & $1.29(0.35$ to 4.75$)$ & 0.70 \\
Exacerbation requiring intravenous & $2.29(0.83$ to 6.34$)$ & 0.11 \\
antibiotics in prior year & & \\
Current therapies & & 0.69 \\
$\quad$ Inhaled antibiotics & $1.25(0.42$ to 3.75$)$ & 0.67 \\
$\quad$ Oral azithromycin & $1.24(0.47$ to 3.29$)$ & \\
\hline BMl, body mass index; MRSA, methicillin-resistant Staphylococcus aureus; MSSA, \\
methicillin-sensitive S. aureus.
\end{tabular}

applied to the panel of proteins with fold difference $>1.5$ between the patients who did versus did not experience an exacerbation within 4 months of stable blood draw to build a MRM-MS classifier. A post hoc sensitivity analysis was conducted to evaluate the impact of altering the fold difference threshold to $>1.25$ and $>1.75$ for protein selection.

Univariable associations between clinical variables and the presence or absence of an exacerbation within 4 months were presented using logistic regression. In multivariable analysis, generalised linear models were applied including all clinical variables (regardless the strength or significance of the association in univariable analysis) to build a clinical classifier. The best-performing clinical classifier was the simplest model (ie, the fewest number of variables) with the highest area under the receiver operating characteristics (ROCs) curve (area under the curve (AUC)). A similar process was performed to identify the best-performing candidate protein biomarker classifier. Ensembling was used to combine classifiers by using average or minimum probabilities from the individual classifiers ${ }^{23}$ to determine whether ensembles improved upon the prognostic performance of the individual classifiers. The performance characteristics of the classifiers were evaluated by leave-one-out cross-validation as described previously $^{24}$ and included AUC, sensitivity, specificity and net reclassification index (NRI). Biomarker score cut-offs were chosen to maximise sensitivity and Youden's index (sensitivity + specificity -1 ) in the discovery cohort. Finally, the classifiers were evaluated in a replication cohort of 34 patients.

\section{RESULTS}

\section{Cohort characteristics}

Characteristics and therapies of the discovery and replication cohorts were similar (table 1). In both cohorts, one-third of participants experienced an exacerbation requiring intravenous antibiotics in the year prior to stable blood draw. A total of 30 (43\%) participants from the discovery cohort and 13 (38\%) participants from the replication cohort were diagnosed with an imminent exacerbation (ie, $<4$ months post-stable blood draw).

\section{Clinical variables to predict exacerbations within 4 months}

In univariable analysis, Pseudomonas aeruginosa infection status was the only clinical variable that was significantly associated with the presence of a pulmonary exacerbation within 4 months of stable blood draw (table 2). There was also a trend towards lower $\mathrm{FEV}_{1} \%$ predicted, lower BMI and a history of at least one pulmonary exacerbation requiring intravenous antibiotics in the prior year among the group of individuals who experienced a pulmonary exacerbation within 4 months of stable blood draw. In multivariable analysis, the best-performing clinical classifier was $\mathrm{FEV}_{1} \%$ predicted alone, with an AUC of 0.55 for the prediction of a pulmonary exacerbation within 4 months (sensitivity $87 \%$, specificity $20 \%$ ) (table 3 ).

\section{Candidate protein biomarkers to predict exacerbations within 4 months}

Based on the discovery cohort, plasma CRP and IL-6 levels were significantly higher in the group of individuals who exacerbated within 4 months of stable blood draw compared with those who did not (table 4). The best-performing individual candidate protein biomarker classifiers were CRP with an AUC of 0.61 (sensitivity 90\%, specificity 35\%) and IL-6 with an AUC of 0.60 (sensitivity 93\%, specificity 28\%) (table 4). In multivariable analysis, various panel combinations of the five-candidate protein biomarkers (including CRP and IL-6 together) did not add to the prognostic performance of CRP and IL-6 individually (data not shown) as CRP and IL-6 were highly correlated with each other $(\mathrm{r}=0.68, \mathrm{p}<0.01)$ (see online supplementary table $\mathrm{S} 2)$.

Table 3 Performance characteristics of the best individual and ensemble classifier panels to predict imminent cystic fibrosis pulmonary exacerbations (ie, $<4$ months post-stable visit)

\begin{tabular}{|c|c|c|c|c|c|c|}
\hline \multirow[b]{2}{*}{ Classifier panel } & \multicolumn{3}{|c|}{ Discovery cohort $(n=70)$} & \multicolumn{3}{|c|}{ Replication cohort $(n=34)$} \\
\hline & AUC & Sensitivity (\%) & Specificity (\%) & AUC & Sensitivity (\%) & Specificity (\%) \\
\hline Best clinical panel* & 0.55 & 90 & 8 & 0.63 & 100 & 10 \\
\hline Best candidate protein panel $\dagger$ & 0.61 & 90 & 35 & 0.55 & 85 & 24 \\
\hline MRM-MS panelł & 0.74 & 90 & 45 & 0.73 & 92 & 48 \\
\hline $\mathrm{CRP}+\mathrm{FEV}_{1} \%$ & 0.61 & 90 & 13 & 0.67 & 92 & 19 \\
\hline MRM-MS+FEV ${ }_{1} \%$ & 0.71 & 90 & 35 & 0.76 & 100 & 24 \\
\hline
\end{tabular}

${ }^{*} \mathrm{FEV}, \%$ predicted alone.

tCRP alone.

‡CRP, peroxiredoxin-2, haemoglobin subunit alpha, carbonic anhydrase 1, CD5, apolipoprotein C-II.

AUC, area under the curve; CRP, C-reactive protein; MRM-MS, multiple reaction monitoring mass spectrometry. 
Table 4 Comparison of candidate plasma protein levels in subjects with versus without an imminent pulmonary exacerbation

\begin{tabular}{llll}
\hline $\begin{array}{l}\text { Candidate } \\
\text { protein* }\end{array}$ & $\begin{array}{l}\text { Fold } \\
\text { differencet }\end{array}$ & $\begin{array}{l}\text { Direction of } \\
\text { differencet }\end{array}$ & p Value \\
\hline CRP & 2.63 & Up & 0.03 \\
IL-6 & 1.67 & Up & 0.02 \\
NEAPC & 1.26 & Up & 0.92 \\
MPO & 1.25 & Up & 0.92 \\
SCD14 & 1.01 & Up & 0.76 \\
\hline
\end{tabular}

*Measured with ELISA except CRP, which was measured with multiple reaction monitoring mass spectrometry.

tPlasma levels of candidate protein among individuals with an imminent pulmonary exacerbation ( $<4$ months) relative to levels among individuals without an imminent pulmonary exacerbation.

CRP, C-reactive protein; IL-6, interleukin-6, MPO, myeloperoxidase; NEAPC, neutrophi elastase antiproteinase complex; sCD14, soluble CD14.

\section{MRM-MS proteomic biomarkers to predict exacerbations within 4 months}

Based on the discovery cohort, 109 peptides (75 proteins) passed quality control and were present in $>75 \%$ of the samples. A number of proteins were differentially abundant in the plasma of individuals who exacerbated within 4 months of stable blood draw compared with those who did not. The 12 proteins that were statistically different between the two groups $(p<0.05)$ or differed by $>1.5$-fold are listed in table 5 . The six proteins with plasma levels that differed by $>1.5$-fold between the two groups were included in the final MRM-MS protein panel that had an AUC of 0.74 (sensitivity 90\%, specificity $45 \%$ ) (table 3). The NRI of the MRM-MS protein panel compared with CRP alone was $10 \%$ and compared with $\mathrm{FEV}_{1} \%$ predicted alone was $28 \%$. The correlation between individual candidate and MRM-MS protein biomarkers is presented in online supplementary table S2, and the association between biomarker score and known determinants of disease severity is presented in online supplementary tables S3, S4 and figure S1. A post hoc sensitivity analysis evaluating the impact of altering

Table 5 List of proteins with differential abundance in the plasma of subjects with versus without an imminent pulmonary exacerbation

\begin{tabular}{lllr}
\hline MRM-MS protein & $\begin{array}{l}\text { Fold } \\
\text { difference }\end{array}$ & $\begin{array}{l}\text { Direction of } \\
\text { difference* }\end{array}$ & p Value \\
\hline CRPt & 2.63 & Up & 0.03 \\
Peroxiredoxin-2† & 2.02 & Down & 0.70 \\
Haemoglobin subunit alpha** $\dagger$ & 1.91 & Down & 0.39 \\
Carbonic anhydrase 1† & 1.67 & Down & 0.06 \\
CD5 $\dagger$ & 1.63 & Down & 0.17 \\
Apolipoprotein C-Il $\dagger$ & 1.59 & Down & 0.03 \\
Protease C1 inhibitor & 1.30 & Down & $<0.01$ \\
Insulin-like growth factor binding protein & 1.26 & Down & $<0.01$ \\
Transthyretin & 1.25 & Down & 0.01 \\
Fibrinogen & 1.23 & Up & 0.01 \\
Serine protease inhibitor & 1.22 & Down & $<0.01$ \\
Albumin & 1.21 & Down & $<0.01$ \\
\hline
\end{tabular}

*Plasma levels of candidate protein among individuals with an imminent pulmonary exacerbation ( $<4$ months) relative to levels among individuals without an imminent pulmonary exacerbation.

tIncluded in final MRM-MS panel.

CRP, C-reactive protein; MRM-MS, multiple reaction monitoring mass spectrometry. the fold difference for protein selection to $>1.25$-fold and $>1.75$-fold demonstrated that the 1.5 -fold threshold resulted in the best performance (see online supplementary table S5).

\section{Ensemble analysis}

The six-protein MRM-MS panel was combined with the bestperforming clinical panel (ie, $\mathrm{FEV}_{1} \%$ predicted alone) and the best-performing candidate protein biomarkers not already included in the MRM-MS panel (ie, IL-6 alone) using ensemble classification. The best ensemble, which involved combining the MRM-MS panel with $\mathrm{FEV}_{1} \%$ predicted, resulted in an AUC of 0.71 (sensitivity 90\%, specificity 35\%) (table 3). Based on an analysis of data that is currently available to CF clinicians, the ensemble of CRP and $\mathrm{FEV}_{1}$ had an AUC of 0.61 (90\% sensitivity, $13 \%$ specificity) (table 3 ). The ROC curves corresponding to the individual and combined panels are shown in figure $2 \mathrm{~A}$.

\section{Classifier replication}

The six-protein MRM-MS biomarker panel was evaluated in the replication cohort and demonstrated an AUC of 0.73, similar to the discovery cohort (AUC 0.74) (table 3). The performance of $\mathrm{CRP}$ alone, $\mathrm{FEV}_{1} \%$ predicted alone, $\mathrm{CRP}+\mathrm{FEV}_{1} \%$ predicted and MRM-MS $+\mathrm{FEV}_{1} \%$ was also evaluated using the replication cohorts, and the results were also similar to those obtained based on the discovery cohort (table 5). The ROC curves of these panels are shown in figure $2 \mathrm{~B}$.

\section{DISCUSSION}

This is the first study to apply MRM-MS to identify bloodbased biomarkers to improve the prediction of impending $\mathrm{CF}$ pulmonary exacerbations. We identified a six-protein panel from plasma that was able to discriminate individuals who are versus are not at imminent ( $<4$ months) risk of an exacerbation with reasonable confidence (AUC 0.74) and replicated our findings using an independent cohort. Importantly, the prognostic performance of the MRM-MS protein panel exceeded clinical variables (eg, $\mathrm{FEV}_{1} \%$ predicted) and individual candidate plasma protein biomarkers (eg, CRP, IL-6), permitting the reclassification of $10-28 \%$ of subjects into correct groups compared with reliance on $\mathrm{CRP}$ or $\mathrm{FEV}_{1} \%$ predicted alone. Furthermore, there was no incremental improvement in performance if traditional markers (eg, $\mathrm{FEV}_{1} \%$ predicted, CRP) were added to the MRM-MS protein panel, suggesting that it might have utility as a standalone test.

In contrast to most prior biomarker discovery studies in CF that have focused on single proteins or molecules to evaluate treatment response or predict/diagnose exacerbations, ${ }^{20}$ we have demonstrated that a panel of six proteins is required to predict a very complex and heterogeneous process such as a pulmonary exacerbation. Although some of the proteins in our panel are biologically plausible in the context of exacerbations, further research is required to follow-up on the significance of individual biomarkers (ie, haemoglobin subunit alpha and carbonic anhydrase 1). Interestingly, five out of six proteins in our panel were less abundant in the plasma of patients with impending exacerbations. While speculative, this raises the possibility that reduced levels of these proteins may confer susceptibility to an exacerbation. For example, the antioxidant enzyme peroxiredoxin- 2 was lower in the plasma of individuals with an impending exacerbation. Imbalance between reactive oxygen species and antioxidant defence can predispose to oxidative stress, and studies have demonstrated that CF pulmonary exacerbations might be either caused or amplified by increased oxidative stress. $^{25} 26$ 

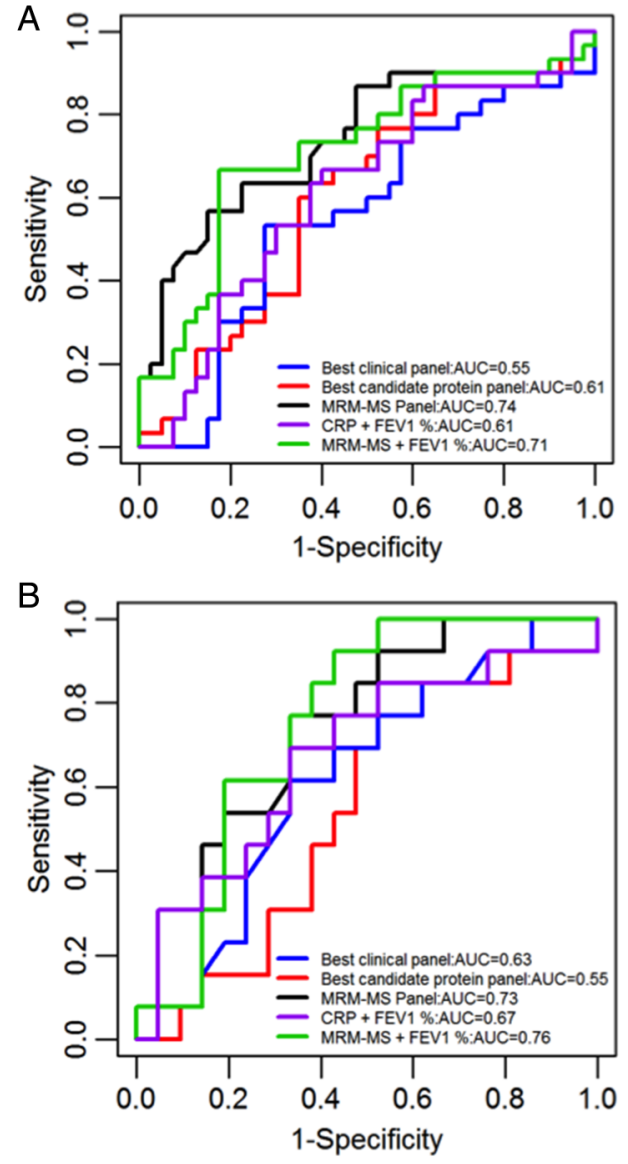

Figure 2 Receiver operating characteristic curves of the individual and ensemble classifiers for the (A) discovery cohort and (B) replication cohort.

The main strengths of our study include the use of a large discovery cohort with clinical characteristics that closely resemble our overall adult CF clinic population. We had very few exclusion criteria in our study design to enhance the generalisability of our findings. We also employed a novel targeted proteomics technology that was recently recognised as Nature's 'Method of the Year' due to its superior multiplexing capabilities. ${ }^{12} \mathrm{~A}$ distinct advantage of this platform is that it does not rely upon affinity-based measurement, which often limits the sensitivity and specificity of antibody-based assays. While there remains no gold standard definition of a pulmonary exacerbation, we used a clinical trial definition that has been endorsed by the EuroCareCF Working Group. ${ }^{16}$

There are a number of important study limitations that should be acknowledged. The peptide/protein library we interrogated with MRM-MS was not unbiased as we used a list of peptides that had been synthesised in prior studies performed in COPD and renal/cardiac allograft rejection. As a result, our biomarker panel cannot be considered truly comprehensive, but this proof-of-concept study provides the opportunity to find new biomarker targets potentially informed by unbiased proteomic techniques or transcriptomics. In this discovery study, we did not adjust our analysis for multiple testing to reduce the risk of missing promising biomarker targets, but this also increased our risk of a type I error. For our primary analysis, we chose to evaluate the biomarker score to predict pulmonary exacerbations within 4 months (ie, prior to the next clinic visit) because we felt this test would be more useful to the clinician. Alternatively, a time-to-event Cox proportional hazards analysis could have been performed to compare time to exacerbation between biomarker score groups and to adjust for potential confounders. While this additional analysis was performed post hoc, our results were generally consistent with our primary analysis and are presented in the online data supplement (see online supplementary table S6 and figure S2). We did not include paediatric patients in this study and therefore our results are not generalisable to this subset of the CF population that is in need for new biomarkers. While we have demonstrated that the MRM-MS panel can discriminate between groups of patients with versus without an imminent exacerbation, we will need to characterise intraindividual variability (ie, signalto-noise ratio) in measurements over time as part of the test validation phase prior to this becoming a clinically validated tool. Lastly, we decided to maximise sensitivity when defining biomarker cut-off scores because a high sensitivity test can reduce the number of missed exacerbation prior to the next clinic visit. As the sensitivity of all three classifiers was excellent $(\geq 90 \%)$, the MRM-MS panel outperformed the candidate protein and clinical classifiers mainly due to improved specificity. The higher specificity of the MRM-MS panel relative to the other classifiers is important clinically because it reduces the false positive rate or the proportion of patients that are predicted to experience an exacerbation when in fact they do not. False positive prognostic test results can lead to the unnecessary initiation of expensive and potentially harmful preventative treatments. With the inherent trade-off between test sensitivity and specificity, the false positive rate might still be considered unacceptably high for use in a clinical setting and therefore the biomarker panel score cut-off could be optimised to increase specificity. A biomarker test to predict exacerbation is unlikely to be perfect because some exacerbations represent stochastic events potentially triggered by an unexpected exposure to a respiratory virus (eg, influenza) for example and thus, may not be predictable.

In summary, we have demonstrated for the first time that MRM-MS can be applied for biomarker discovery in CF to predict imminent pulmonary exacerbations. While our discovery phase results are encouraging, the MRM-MS panel is not yet ready for use as a clinically validated tool as our findings must be placed within the scope of the biomarker development process as outlined by the Institute of Medicine. ${ }^{27}$ MRM-MS is a platform that has been used in the clinical lab to measure small molecules (eg, hormones, metabolites, drugs) but requires further research and optimisation prior to its application in the clinical lab for routine peptide/protein analysis. ${ }^{12} 28$ Future research directions will include replication in an external cohort, analytical and clinical/biological validation, and evaluation in a clinical utility study.

\section{Author affiliations}

${ }^{1}$ Centre for Heart Lung Innovation, University of British Columbia and St. Paul's Hospital, Vancouver, British Columbia, Canada

${ }^{2}$ Division of Respiratory Medicine, Department of Medicine, University of British Columbia, Vancouver, British Columbia, Canada

${ }^{3}$ PRevention Of Organ Failure (PROOF) Centre of Excellence, Vancouver, British Columbia, Canada

${ }^{4}$ Department of Computer Science, University of British Columbia, Vancouver, British Columbia, Canada

Correction notice This article has been corrected since it was published Online First. The provenance and peer review statement has been corrected.

Acknowledgements We thank our patients for their participation. We would also like to thank Lynda Lazosky, Eri Flores, Sheena Tam, Yeni Oh and David Ngan for their assistance with patient recruitment and sample processing. 
Contributors BSQ was involved in the study concept and design, data analysis and manuscript preparation. DLYD, ZH and RTN were involved in the data analysis and manuscript preparation. DDS, PGW, SFPM and SJT were involved in the study concept and design and revising it critically for important intellectual content. All authors are agreeable to the final version to be published and accept accountability for the integrity of all aspects of the work.

Funding The British Columbia Lung Association, Providence Health Care Research Institute and Cystic Fibrosis Canada provided grant support for this study.

Competing interests None.

Ethics approval University of British Columbia-Providence Health Care (UBC-PHC) Research Institute Ethics Board.

Provenance and peer review Not commissioned; externally peer reviewed.

\section{REFERENCES}

1 Sanders DB, Bittner RC, Rosenfeld M, et al. Pulmonary exacerbations are associated with subsequent FEV1 decline in both adults and children with cystic fibrosis. Pediatr Pulmonol 2011;46:393-400.

2 Sanders DB, Bittner RC, Rosenfeld $M$, et al. Failure to recover to baseline pulmonary function after cystic fibrosis pulmonary exacerbation. Am J Respir Crit Care Med 2010;182:627-32.

3 Yi MS, Tsevat J, Wilmott RW, et al. The impact of treatment of pulmonary exacerbations on the health-related quality of life of patients with cystic fibrosis: does hospitalization make a difference? J Pediatr 2004;144:711-18.

4 Britto MT, Kotagal UR, Hornung RW, et al. Impact of recent pulmonary exacerbations on quality of life in patients with cystic fibrosis. Chest 2002;121:64-72.

5 Liou TG, Adler FR, Fitzsimmons SC, et al. Predictive 5-year survivorship model of cystic fibrosis. Am J Epidemiol 2001;153:345-52.

6 Mayer-Hamblett N, Rosenfeld M, Emerson J, et al. Developing cystic fibrosis lung transplant referral criteria using predictors of 2-year mortality. Am J Respir Crit Care Med 2002;166(12 Pt 1):1550-5.

7 Cystic Fibrosis Foundation Patient Registry. 2012 Annual Data Report to the Center Directors. Bethesda, Maryland: Cystic Fibrosis Foundation, 2013.

8 Mogayzel PJ Jr, Naureckas ET, Robinson KA, et al. Cystic fibrosis pulmonary guidelines. Chronic medications for maintenance of lung health. Am J Respir Crit Care Med 2013;187:680-9.

9 Block JK, Vandemheen KL, Tullis $\mathrm{E}$, et al. Predictors of pulmonary exacerbations in patients with cystic fibrosis infected with multi-resistant bacteria. Thorax 2006;61:969-74.

10 Sawicki GS, Ayyagari R, Zhang J, et al. A pulmonary exacerbation risk score among cystic fibrosis patients not receiving recommended care. Pediatr Pulmonol 2013;48:954-61.

11 Wheelock CE, Goss VM, Balgoma D, et al. Application of 'omics technologies to biomarker discovery in inflammatory lung diseases. Eur Respir J 2013;42:802-25.
12 Gillette MA, Carr SA. Quantitative analysis of peptides and proteins in biomedicine by targeted mass spectrometry. Nat Methods 2013;10:28-34.

13 Chaussabel D, Pascual V, Banchereau J. Assessing the human immune system through blood transcriptomics. BMC Biol 2010;8:84.

14 Farrell PM, Rosenstein BJ, White TB, et al. Guidelines for diagnosis of cystic fibrosis in newborns through older adults: cystic Fibrosis Foundation consensus report. J Pediatr 2008;153:S4-14.

15 Fuchs HJ, Borowitz DS, Christiansen DH, et al. Effect of aerosolized recombinant human DNase on exacerbations of respiratory symptoms and on pulmonary function in patients with cystic fibrosis. The Pulmozyme Study Group. N Engl J Med 1994;331:637-42.

16 Bilton D, Canny G, Conway S, et al. Pulmonary exacerbation: towards a definition for use in clinical trials. Report from the EuroCareCF Working Group on outcome parameters in clinical trials. J Cyst Fibros 2011;10(Suppl 2):S79-81.

17 Standardization of spirometry-1987 update. Statement of the American thoracic society. Am Rev Respir Dis 1987;136:1285-98.

18 Kuzyk MA, Parker CE, Domanski D, et al. Development of MRM-based assays for the absolute quantitation of plasma proteins. Methods Mol Biol 2013;1023:53-82.

19 Kuzyk MA, Smith D, Yang J, et al. Multiple reaction monitoring-based, multiplexed, absolute quantitation of 45 proteins in human plasma. Mol Cell Proteomics 2009;8:1860-77.

20 Shoki AH, Mayer-Hamblett N, Wilcox PG, et al. Systematic review of blood biomarkers in cystic fibrosis pulmonary exacerbations. Chest 2013;144:1659-70.

21 Hollander Z, Lazarova M, Lam KK, et al. Proteomic biomarkers of recovered heart function. Eur J Heart Fail 2014;16:551-9.

22 Smyth GK. Linear models and empirical bayes methods for assessing differential expression in microarray experiments. Stat Appl Genet Mol Biol 2004;3: Article3.

23 Gunther OP, Chen V, Freue GC, et al. A computational pipeline for the development of multi-marker bio-signature panels and ensemble classifiers. BMC Bioinformatics 2012;13:326.

24 Lin D, Cohen Freue G, Hollander Z, et al. Plasma protein biosignatures for detection of cardiac allograft vasculopathy. J Heart Lung Transplant 2013;32:723-33.

25 Reid DW, Misso N, Aggarwal S, et al. Oxidative stress and lipid-derived inflammatory mediators during acute exacerbations of cystic fibrosis. Respirology 2007;12:63-9.

26 McGrath LT, Mallon P, Dowey L, et al. Oxidative stress during acute respiratory exacerbations in cystic fibrosis. Thorax 1999;54:518-23.

27 Omenn GS. On best practices: the Institute of Medicine scheme for developing, validating, and demonstrating clinical utility of omics-based diagnostic and predictive tests. Proteomics Clin Appl 2013;7:748-55.

28 Grant RP, Hoofnagle AN. From lost in translation to paradise found: enabling protein biomarker method transfer by mass spectrometry. Clin Chem 2014;60:941-4. 